Math. Model. Nat. Phenom.

Vol. 6, No. 5, 2011, pp. 333-349

DOI: $10.1051 / \mathrm{mmnp} / 20116513$

\title{
Blood Flow Simulation in Atherosclerotic Vascular Network Using Fiber-Spring Representation of Diseased Wall
}

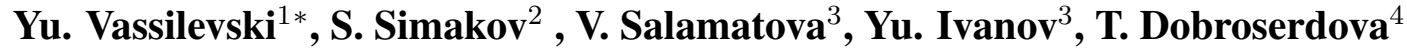 \\ ${ }^{1}$ Institute of Numerical Mathematics RAS, 119333, Moscow, 8 Gubkina St., Russia \\ ${ }^{2}$ Moscow Institute of Physics and Technology, 141700, Dolgoprudny, 9 Instituskii Lane, Russia \\ ${ }^{3}$ Scientific Educational Center of Institute of Numerical Mathematics RAS \\ 119333, Moscow, 8 Gubkina St., Russia \\ ${ }^{4}$ Moscow State University, 119991, Moscow, Leninskie Gory, Russia
}

\begin{abstract}
We present the fiber-spring elastic model of the arterial wall with atherosclerotic plaque composed of a lipid pool and a fibrous cap. This model allows us to reproduce pressure to crosssectional area relationship along the diseased vessel which is used in the network model of global blood circulation. Atherosclerosis attacks a region of systemic arterial network. Our approach allows us to examine the impact of the diseased region onto global haemodynamics.
\end{abstract}

Key words: atherosclerosis, mathematical modeling, blood flow, arterial wall AMS subject classification: 35Q92, 76Z05, 65M06, 65M25

\section{Introduction}

Atherosclerosis is an inflammatory process in the vessel wall. Atherosclerotic plaque is a flexible formation with complex structure. Important aspect of atherosclerosis is that it attacks a region of systemic arterial network rather than single artery. Therefore, it is a global process evolving in a connected set of arteries.

Many works consider atherosclerosis as a local process studying the development of single atherosclerotic plaque, its rupture and its impact onto the blood flow in the separate vessel. Reaction-diffusion [6], layer-specific composite wall material under static load [8, 4, 5] and re-

${ }^{*}$ Corresponding author. E-mail: vasilevs@ dodo.inm.ras.ru 
cently fluid-structure interaction (FSI) [7] methodologies has been widely used. These approaches operate with dynamics of spatially distributed systems. Feasibility of such models is highly improved by using patient specific data obtained from magnetic resonance imaging and other clinical diagnosis techniques. Nevertheless, it is still impossible to use such models for simulating the regional blood flow changes in atherosclerotic vascular network due to computational power restriction, complex geometry of the diseased region and ill-defined clinical parameters rarely accessible for such large-scale simulations.

The presented approach allows us to examine regional blood flow in atherosclerotic vascular network as well as in the whole vascular network with several diseased regions. It exploits the idea of coupling the model of network blood circulation $[2,12,13,15]$ and the model of elastic vessel wall response $[3,11,14]$. The wall model considers vessel wall material as a fiber-reinforced fluid. Although this approach can be used for modelling an arbitrary incompressible linearly elastic material [9], we restrict ourselves by the particular case of the three-layer cylindrical tube. We propose the fiber-spring elastic model of the arterial wall with atherosclerotic plaque composed of a lipid pool and a fibrous cap.

Applying the static load to vessel wall represented by the proposed fiber-spring elastic model, we obtain the wall state equation, i.e. the pressure to cross-sectional area relationship along the diseased vessel. This relation is used in the network model of global blood circulation [12] instead of the conventional wall state equation. The modification of the wall state equation is used for all diseased vessels. This allows us to examine the impact of diseased regions onto haemodynamics. Numerical recovery of the wall state equation was initially proposed for the simulation of the impact of an intravenous implant [16].

\section{Fiber-spring representation of the atherosclerotic vessel}

We consider the atherosclerotic blood vessel as a three-layer circular cylindrical shell inflated by internal pressure. The strain in the axial direction is assumed to be negligible. Strictly speaking this assumption is valid for lengthy stenotic regions under axysimmetric loading but we extend it for all cases considered in this work. Similar plane strain approximation has been used by Cheng et. al. [1]. The internal and external layers are thin-walled cylindrical shells which represent the fibrous cap of the atherosclerotic plaque and the vessel wall, respectively. The middle layer represents the lipid pool of the atherosclerotic plaque. The arterial wall and the atherosclerotic plaque is assumed to be isotropic and linearly elastic. Every layer is modelled as a set of fibers. Each fiber is described by two independent cylindrical coordinates.

\subsection{Fiber model of the fibrous cap and the arterial wall}

The elastic models of the fibrous cap and the arterial wall are based on the assumption of thinwalled circular cylindrical shell which neglects the radial stress $\sigma_{r}$. We use the independent ring model [2] which allows us to ignore the axial stress $\sigma_{x}$ and to use the following relation between 
circumferential stress $\sigma_{\theta}$ and circumferential strain $\varepsilon_{\theta}$ :

$$
\sigma_{\theta}=\frac{E}{1-\nu^{2}} \varepsilon_{\theta},
$$

where $E, \nu$ are Young's modulus and Poisson's ratio of the cylindrical shell, respectively. Hereinafter $(r, \theta, x)$ denotes the cylindrical coordinate system.

Using the approach described in [11], we imitate the response of the elastic surface to a deformation as the response of fibers collection to the same deformation. Let $\vec{X}(s, t)$ represent the position of the fiber points in space, where Lagrange coordinate $s$ is the arc length of the fiber in the unstressed state. We define the tension force $T(s, t)$ in the fiber as

$$
T=\left\{\begin{array}{l}
T_{*}\left(\left|\frac{\partial \vec{X}}{\partial s}\right|-1\right), \quad\left|\frac{\partial \vec{X}}{\partial s}\right|>1 \\
0, \\
\left|\frac{\partial \vec{X}}{\partial s}\right| \leq 1
\end{array}\right.
$$

where $T_{*}$ is the elastic modulus of the fiber. The value $T_{*}$ is the same for fibers of the same kind.

The local force density is given by the expression [14]

$$
\vec{f}=\frac{\partial}{\partial s}(T \vec{\tau})
$$

where $\vec{\tau}$ is the unit tangent vector

$$
\vec{\tau}=\frac{\partial \vec{X}}{\partial s} /\left|\frac{\partial \vec{X}}{\partial s}\right| .
$$

In the case of axisymmetric problem we can present the middle surface of the cylindrical shell as a collection of independent ring fibers. The tension force $T$ is the circumferential stress $\sigma_{\theta}$ and thus the fiber elastic modulus satisfies $T_{*}=E /\left(1-\nu^{2}\right)$.

Let $N$ be the number of computational nodes on the fiber, $X_{k}$ be the coordinates of the $k$ th node, $k=1, \ldots, N, \Delta s$ be the distance between neighboring nodes along the non-deformed fiber. We assume that $\Delta s$ will be the same for fibers of the same kind. In accordance with formulas (2.1), (2.3) we discretize $T$ and $\vec{\tau}$ :

$$
\begin{gathered}
T_{k+1 / 2}=T_{*}\left(\frac{\left|\vec{X}_{k+1}-\vec{X}_{k}\right|}{\Delta s}-1\right), \frac{\left|\vec{X}_{k+1}-\vec{X}_{k}\right|}{\Delta s}>1 \\
\vec{\tau}_{k+1 / 2}=\frac{\vec{X}_{k+1}-\vec{X}_{k}}{\left|\vec{X}_{k+1}-\vec{X}_{k}\right|} .
\end{gathered}
$$

The discrete elastic force at the $k$ th node is defined as

$$
\overrightarrow{f_{k}}=\frac{T_{k+1 / 2} \vec{\tau}_{k+1 / 2}-T_{k-1 / 2} \vec{\tau}_{k-1 / 2}}{\Delta s} .
$$

The above formulas set up our numerical fiber elastic model of the response to the deformation. 


\subsection{Spring model of the lipid pool}

The elastic model of the lipid pool is based on its representation by a set of radial springs. In order to estimate spring stiffness, we use the solution of the deformation problem for the isotropic cylinder $\left(a_{1} \leq r \leq b_{1}\right)$ under internal pressure $p_{1}$ and fixed support boundary condition on the external cylindrical surface. The problem is solved by using the hypothesis of linear elasticity theory. In this case the radial displacement $u^{(c)}(r)$ and radial stress $\sigma_{r}^{(c)}$ are given by

$$
\begin{gathered}
u^{(c)}(r)=-\frac{p_{1} a_{1}^{2}\left(1-2 \nu_{c}\right)\left(1+\nu_{c}\right)}{E_{c}\left(a_{1}^{2}+\left(1-2 \nu_{c}\right) b_{1}^{2}\right)}\left(r-\frac{b_{1}^{2}}{r}\right), \\
\sigma_{r}^{(c)}(r)=-\frac{p_{1} a_{1}^{2}}{\left(a_{1}^{2}+\left(1-2 \nu_{c}\right) b_{1}^{2}\right)}\left(1-\left(2 \nu_{c}-1\right) \frac{b_{1}^{2}}{r^{2}}\right),
\end{gathered}
$$

where $E_{c}, \nu_{c}$ are Young's modulus and Poisson's ratio of the cylinder, respectively. Equations (2.7), (2.8) define implicitly the relation between radial displacement and radial stress. We define the relative radial extension $\Delta=\left(u\left(b_{1}\right)-u\left(a_{1}\right)\right) /\left(b_{1}-a_{1}\right)$ and rewrite (2.7), (2.8) expressing the radial stress via the non-constant elastic modulus of the spring $E_{s p}(r)$

$$
\begin{gathered}
\sigma_{r}^{(c)}(r)=E_{s p}(r) \Delta \\
E_{s p}(r)=\frac{a_{1} E_{c}}{\left(a_{1}+b_{1}\right)\left(1-2 \nu_{c}\right)\left(1+\nu_{c}\right)}\left(1+\left(1-2 \nu_{c}\right) \frac{b_{1}^{2}}{r^{2}}\right) .
\end{gathered}
$$

Therefore, the spring imitating the elastic response of the lipid pool to the internal pressure must have the elastic modulus dependent on the position of the fibrous cap.

\subsection{Verification of the fiber-spring model}

To summarize, we consider the diseased vessel as a three-layer cylindrical shell whose interior (fibrous cap) and exterior (arterial wall) layers are thin and are represented as the collection of ring fibers with elastic modulus $E /\left(1-\nu^{2}\right)$. The intermediate layer is modelled by radial springs with the non-constant elasticity modulus defined by (2.9).

We compare the numerical solution of the deformation problem for the three-layer cylindrical shell under internal pressure $p$ using the fiber-spring model with the analytic solution in the case of linear elasticity. We denote by $a-h_{1} / 2 \leq r \leq a+h_{1} / 2$ the first layer (the fibrous cap), by $a+h_{1} / 2<r \leq b-h_{2} / 2$ the second layer (the lipid pool), and by $b-h_{2} / 2<r \leq b+h_{2} / 2$ the third layer (the arterial wall). Then the radial displacement and the stresses of each of the layers are given by

$$
\begin{gathered}
u^{(i)}(r)=C_{1}^{(i)} r+\frac{C_{2}^{(i)}}{r} \\
\sigma_{r}^{(i)}(r)=\frac{E_{i}}{\left(1+\nu_{i}\right)\left(1-2 \nu_{i}\right)}\left(C_{1}^{(i)}-\frac{C_{2}^{(i)}}{r^{2}}\right), \sigma_{\theta}^{(i)}(r)=\frac{E_{i}}{\left(1+\nu_{i}\right)\left(1-2 \nu_{i}\right)}\left(C_{1}^{(i)}+\frac{C_{2}^{(i)}}{r^{2}}\right),
\end{gathered}
$$




\begin{tabular}{|c|c|c|c|c|c|}
\hline & $p, \mathrm{KPa}$ & $u_{a n}(a), \mathrm{cm}$ & $u_{f s}(a), \mathrm{cm}$ & $u_{a n}(b), \mathrm{cm}$ & $u_{f s}(b), \mathrm{cm}$ \\
\hline$a=0.9192 \mathrm{~cm}$ & 5 & 0.0316 & 0.0321 & 0.0206 & 0.0226 \\
\cline { 2 - 6 } $\begin{array}{c}a=1.3 \mathrm{~cm} \\
h_{1}=0.1 \mathrm{~cm}\end{array}$ & 10 & 0.0506 & 0.0515 & 0.0330 & 0.0365 \\
$h_{2}=0.1 \mathrm{~cm}$ & 13 & 0.0632 & 0.0645 & 0.0413 & 0.0457 \\
\cline { 2 - 6 } & 15 & 0.0948 & 0.0840 & 0.0536 & 0.0596 \\
\hline
\end{tabular}

Table 1: Comparison between analytic displacements $u_{a n}$ and numerical displacements $u_{f s}$ due to the fiber-spring model.

where $E_{i}, \nu_{i}$ are Young's modulus and Poisson's ratio of the $i$-th layer respectively, $i=1,2,3$ defines the number of the layer. The constants $C_{1}^{(i)}, C_{2}^{(i)}$ can be obtained from boundary conditions and continuity conditions of radial displacement and radial stress. If $a=0.9192 \mathrm{~cm}, b=1.3 \mathrm{~cm}$, $h_{1}=h_{2}=0.1 \mathrm{~cm}$ then

$$
\begin{gathered}
C_{1}^{(1)}=0.237910^{-4} p, C_{2}^{(1)}=0.561110^{-7} p, \\
C_{1}^{(2)}=-0.776710^{-4} p, C_{2}^{(2)}=0.656410^{-7} p, \\
C_{1}^{(3)}=0.113510^{-4} p, C_{2}^{(3)}=0.517310^{-7} p .
\end{gathered}
$$

The numerical solution of the fiber-spring model is obtained for the following values of elastic constants: $E_{1}=1000 \mathrm{KPa}, E_{2}=1 \mathrm{KPa}, E_{3}=50 \mathrm{KPa}, \nu_{1}=\nu_{2}=\nu_{3}=0.48$. The radial displacements of the middle surfaces $r=a$ and $r=b$ are summarized in Table 1 where $u_{a n}(r)$ stands for the analytic solution and $u_{f s}(r)$ stands the numerical solution obtained by the fiberspring model. We can see that the error at internal radius $r=a$ is less than $2 \%$, in the case $r=b$ the error is less than $11 \%$. The last deviation can be explained by limitations of the linear elasticity theory and different boundary conditions in the problem for the estimation of the spring stiffness and in the problem used in the verification.

\section{Network blood circulation model}

\subsection{Basic equations}

In the network circulation model the domain is a set of 1D flexible channels connected in a closed network consisting of four parts (pulmonary and systemic arteries and veins). The main attention of this work is focused on systemic arteries as the most attractive for the atherosclerotic plaques. Pulmonary circulation is excluded from our consideration.

Blood flow is considered as pulsating flow of incompressible fluid streaming through the network of elastic tubes (vessels). For every vessel we watch out for mass and momentum change by 
the following $1 \mathrm{D}$ balance equations in the characteristic form [12]:

$$
\begin{aligned}
\partial S / \partial t+\partial(S u) / \partial x & =\varphi\left(t, x, S, u, \chi_{i}\right), \\
\partial u / \partial t+\partial\left(u^{2} / 2+p / \rho\right) / \partial x & =\psi\left(t, x, S, u, \chi_{i}\right),
\end{aligned}
$$

where $t$ is time, $x$ is coordinate along the vessel, $\rho$ is blood density, $S(t, x)$ is vessel cross section area, $u(t, x)$ is linear flow velocity averaged over the vessel area at coordinate $x, p$ is pressure (relative to the atmospheric), $\varphi$ is mass inflow/outflow (e.g. due to the damage of the vessel wall or blood transfusion), $\psi$ is resulting force applied to the vessel (e.g. gravity, friction, etc.), $\left\{\chi_{i}\right\}_{i=1}^{I}$ is a set of constants. In this work we use

$$
\varphi\left(t, x, S, u, \chi_{i}\right)=0
$$

and

$$
\psi\left(t, x, S, u, \chi_{i}\right)=-16 \mu u \frac{\eta(\hat{S})}{\hat{S}},
$$

where $\hat{S}=S / \bar{S} ; \bar{S}$ is cross section area under zero transmural pressure $p(S)-p_{*}$ and zero velocity; $p_{*}$ is the pressure in the surrounding tissues; $\mu$ is friction factor;

$$
\eta(\hat{S})=\left\{\begin{array}{l}
2, \hat{S} \geqslant 1 \\
\hat{S}+\frac{1}{\hat{S}}, \hat{S}<1 .
\end{array}\right.
$$

Elastic properties of the vessel wall are described by the wall state equation defining the dependence of transmural pressure $p-p_{*}$ on the vessel cross section area $S$

$$
p(S)-p_{*}=\rho c_{0}^{2} f(S),
$$

where $c_{0}$ is the rate of small disturbance propagation in the vessel wall. The wall state equation is discussed in the next section.

The vessels are connected with each other at the nodes and with the heart inlets/outlets through the boundary conditions set that is formed by Poiseuille's pressure drop conditions, mass balance equation combined with the appropriate compatibility condition for (3.1),(3.2)

$$
\begin{gathered}
p_{k}\left(S_{k}\left(t, \tilde{x}_{k}\right)\right)-p_{\text {node }}^{l}(t)=\varepsilon_{k} R_{k}^{l} S_{k}\left(t, \tilde{x}_{k}\right) u_{k}\left(t, \tilde{x}_{k}\right), k=k_{1}, k_{2}, \ldots, k_{M}, \\
\sum_{k=k_{1}, k_{2}, \ldots, k_{M}} \varepsilon_{k} S_{k}\left(t, \tilde{x}_{k}\right) u_{k}\left(t, \tilde{x}_{k}\right)=0,
\end{gathered}
$$

where $l$ is node's index, $k$ is vessel index, $k_{1}, k_{2}, \ldots, k_{M}$ and $M$ are the indexes and the number of the vessels meeting at the node; $p_{\text {node }}^{l}(t)$ is pressure at the vessels junction point; $R_{k}^{l}$ is hydraulic resistance for the flow from the $k$-th vessel to the $l$-th node. For the vessels incoming into a node we set $\varepsilon_{k}=1, \tilde{x}_{k}=L_{k}$, for the outgoing vessels we set $\varepsilon_{k}=-1, \tilde{x}_{k}=0$. At the heart junction nodes the product $S_{k}(t, x) u_{k}(t, x)$ in (3.7),(3.8) is replaced with volumetric flow to/from the appropriate chamber $Q_{k}$. 
The structure of the arterial network of systemic circulation is shown in the left picture of Fig. 1. It was reconstructed on the basis of [2]. Some parts of the network were refined and additional vessels were added to adjust the total blood in the network to the appropriate physiological value. The structure of the venous network of systemic circulation is almost the mirrored image of the arterial part and thus is not presented here. The difference is that initial diameters of the veins are nearly 2 times greater than their arterial counterparts and $c_{0}$ is 1.5 times smaller. In some numerical experiments studying qualitative behaviour we also have considered a simplified counterpart of the arterial network shown in the right picture of Fig. 1.
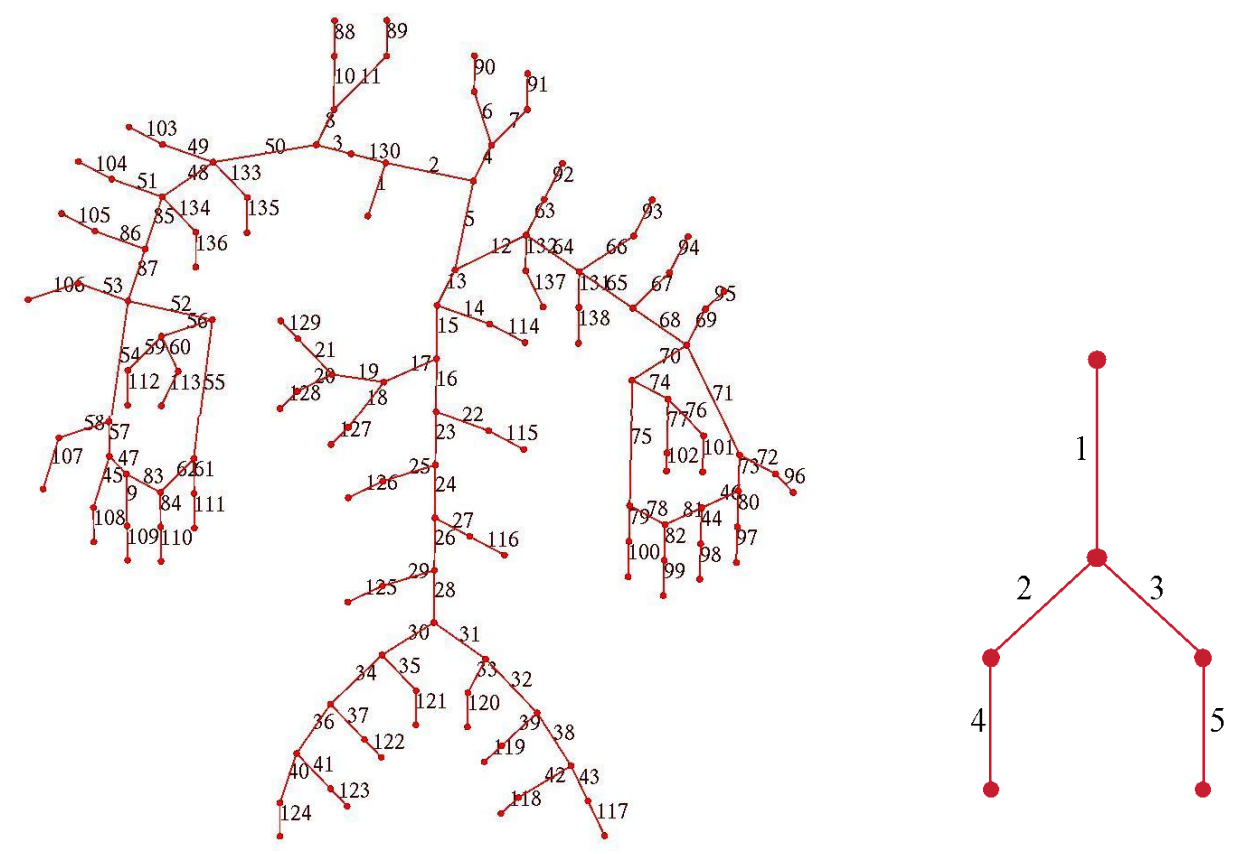

Figure 1: Arterial network structure (left) and its simplified counterpart (right).

\subsection{Wall state equations for healthy and diseased vessels}

In this section we present our approach to deriving the wall state equation (3.6). In general, function $f(S)$ depends on the type and state of the vessel wall (elastic or muscular type of the wall, installed endovascular devices, atherosclerotic plaque, occlusion, etc.). The plot of $f$ has $S$-like shape [10] for the healthy vessel. In this work for the most (healthy) vessels we use expressions [12]

$$
f(S)= \begin{cases}\exp (S / \bar{S}-1)-1, & S>\bar{S}, \\ \ln (S / \bar{S}), & S \leqslant \bar{S} .\end{cases}
$$

Vessels damaged by atherosclerosis have different wall state equation. We note that the numerical fiber model of the elastic healthy wall recovers function (3.9) with sufficient accuracy under the assumption that the transmural pressure is counterbalanced by the elastic force of the fiber deformation. 
We use the fiber-spring model to define the wall state equation for the vessels damaged by atherosclerosis. We consider three types of plaque geometry that are shown in Figs. 2, 3, 4. All three types are formed by the same outer and different inner surfaces. The inner surface corresponds to the fibrous cover of the plaque. The surfaces are represented by sets of fibers which are connected with springs as shown in Figs. 2, 3, 4. The first type (Fig. 2) represents the connected coaxial cylindrical shells that corresponds to the lengthy plaques which are uniformly distributed over the vessel wall. The second type (Fig. 3) is formed by the interior axial symmetric surface narrowing at the center of the vessel. The third type (Fig. 4) is similar to the second one but the axial symmetry is violated.

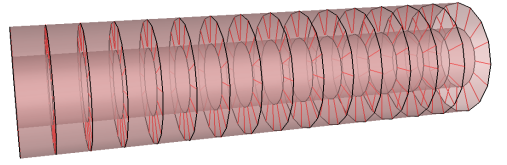

Figure 2: Geometry of the plaque, type 1.

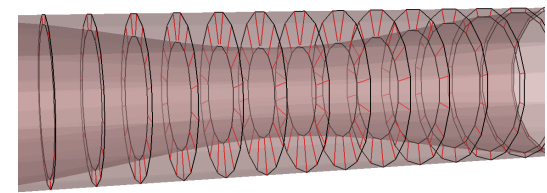

Figure 3: Geometry of the plaque, type 2.

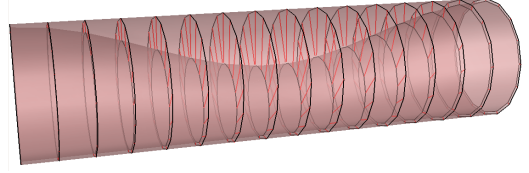

Figure 4: Geometry of the plaque, type 3.

A set of preliminary simulations was carried out for every geometry. The static inflation pressure load was applied at some range and the cross-sectional area was calculated along the vessel. The results shown in Fig. 5 provide the example of $p(S)-p_{*}$ functions for the diseased vessel of types 2 and 3 and minimal lumen $30 \%$ that substitutes corresponding wall state equation (3.6) for the healthy vessel. This function differs for the cross-sections along the $x$ coordinate due to inhomogeneity of the inner wall near the center of the vessel. The type 1 plaque simulations produce the wall state function $p(S)-p_{*}$ remaining the same along the $x$ coordinate since this type introduces homogeneous geometry of the inner plaque cover along the vessel. We note that atherosclerotic plaques of types 2 and 3 produce $p(S, x)-p_{*}$ function that is naturally fitted to the network circulation model. Analogous simulations were performed also for the cases of minimal lumen $10 \%$ and $50 \%$.

Details on numerical implementation of the network blood circulation model can be found in [12] with some improvements introduced in [16]. 

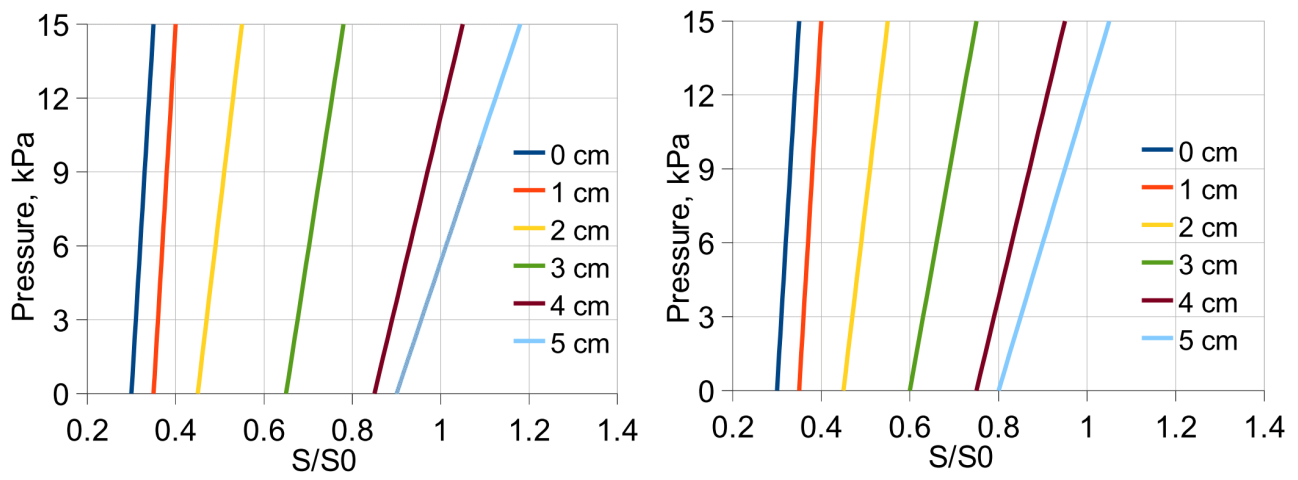

Figure 5: Wall state dependencies for the plaque of type 2 (left) and type 3 (right). The minimal lumen is $30 \%$. The index of each curve corresponds to the distance (in $\mathrm{cm}$ ) to the minimal cross section. $S_{0}$ denotes the cross-sectional area of the relaxed healthy artery.

\section{Results}

At first we present our results of the 1D haemodynamics simulation on the simplified arterial network structure shown in the right panel of Fig. 1. The vessel with number 2 is assumed to be diseased. Geometric characteristics (length $l$ and diameter $d$ ) for this structure are presented in Table 2.

\begin{tabular}{|c|c|c|c|c|c|}
\hline parameter $\backslash$ vessel & 1 & 2 & 3 & 4 & 5 \\
\hline$l, \mathrm{~cm}$ & 10 & 20 & 20 & 5 & 5 \\
$d, \mathrm{~cm}$ & 2 & 1.6 & 1.6 & 2 & 2 \\
\hline
\end{tabular}

Table 2: Geometric characteristics of arteries in the simplified network structure.

We compare the impact of atherosclerotic plaque at artery 2 with the minimal lumen (along $x$ axis, in the unstressed state) $50 \%, 30 \%, 10 \%$ considering different types of the plaque model. The pressure and velocity profiles for the plaque models 1 and 2 are shown in Figs. 6-9. The comparison of the different plaque models is presented in Figs. 10, 11. The results for the plaque models 2 and 3 are similar. $x$ coordinate counts off the beginning of the vessel at every figure.

In the case of plaque model 1 we notice the remarkable velocity gradient near the endpoints of artery 2 with the minimal lumen 10\% (see Fig. 7). We ascribe the velocity gradient to the increased impact of the viscosity. In all cases of the lumen we observe the difference between the velocity profiles for the diseased and healthy vessel. Thus, the lengthy plaques are haemodinamically substantial and can be determined by means of ultra Doppler measurements.

In the case of plaque model 2 we observe the distinct pressure drop in artery 2 with the minimal lumen $10 \%$ (see Fig. 8). This drop results in the development of a stagnation zone before the minimal cross-section (see Fig. 9). The stagnation zone is potentially attractive for the upstream growth of the plaque.

The comparison of Figs. 6, 7 and Figs. 8, 9 reveals substantial difference of impacts of plaques 1 and 2 on haemodynamics. Luminal narrowing causes both pressure and velocity increase. How- 

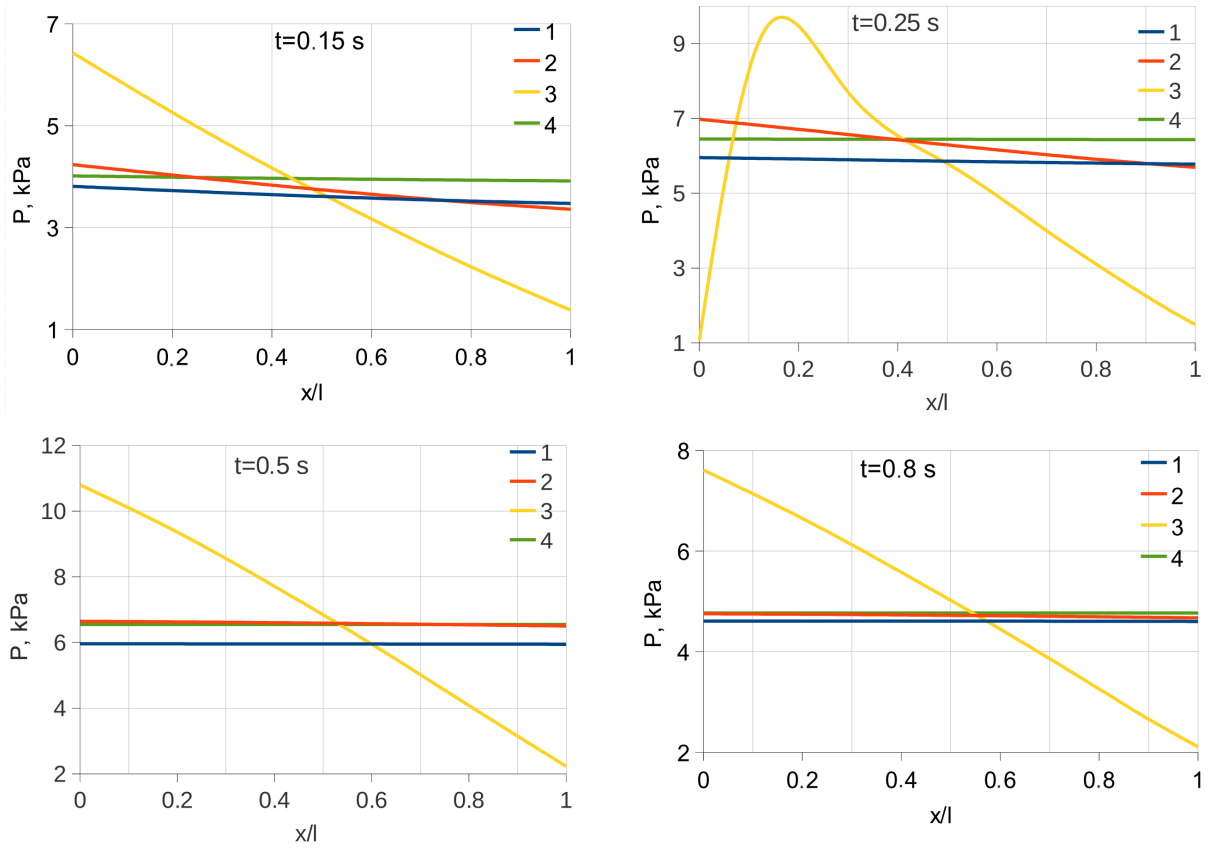

Figure 6: Pressure profiles in artery 2 with plaque 1 ( $1-50 \%$ lumen, $2-30 \%$ lumen, $3-10 \%$ lumen, 4 - healthy vessel) at $0.15,0.25,0.5,0.8$ seconds after the beginning of the cardiac cycle.

ever, for plaque model 2 the velocity at the inlet and outlet is almost the same as that under the healthy conditions. Thus, in spite of remarkable changes in the profiles inside artery 2, only the smallest plaque lumen can provide a noticeable impact on the flow in neighbouring vessels. Moreover, in practice it is hard to observe the essential change of pressure. This is the reason why it is difficult to detect the plaques at some stages of their development.

The comparative analysis (Figs. 10,11) of the impact of the plaque geometry with the same minimal lumen $30 \%$ shows that the velocity profiles for plaques 2 and 3 practically coincide and can be separated from the profiles of the healthy vessel or vessel with plaque 1 at different stages of cardiac cycle. Only the pressure profile allows us to identify the geometric model of the plaque.

Vascular network simulations allow us to access blood flow redistribution in the region of the diseased vessel. In order to demonstrate this, we consider the systemic circulation with arterial network shown in the left picture of Fig. 1. The vessel designated with index 49 is supposed to be diseased and is assigned to plaque 1 with minimal lumen 10\%. It has the following parameters: $l=$ $5 \mathrm{~cm}, d=0.8 \mathrm{~cm}$. Its pressure to cross-section relationship was generated by the fiber-spring model. The remaining vessels of the network have wall state equation (3.6), (3.9) with the parameter $c_{0}=900 \mathrm{~cm} / \mathrm{s}$ for the arteries and $c_{0}=450 \mathrm{~cm} / \mathrm{s}$ for the veins.

We compare regional blood flow characteristics for artery 49 in healthy and diseased states. Its neighbors may be split into distal $(48,133)$ and proximal vessels $(50,103)$. Time dependencies of the pressure and velocity are measured at the central point of corresponding vessel and are shown in Figs. 12-16. The dominant negative velocity value in Fig. 16 is due to the positive orientation of $x$ coordinate that points to the junction point. All other examined vessels are directed from the 

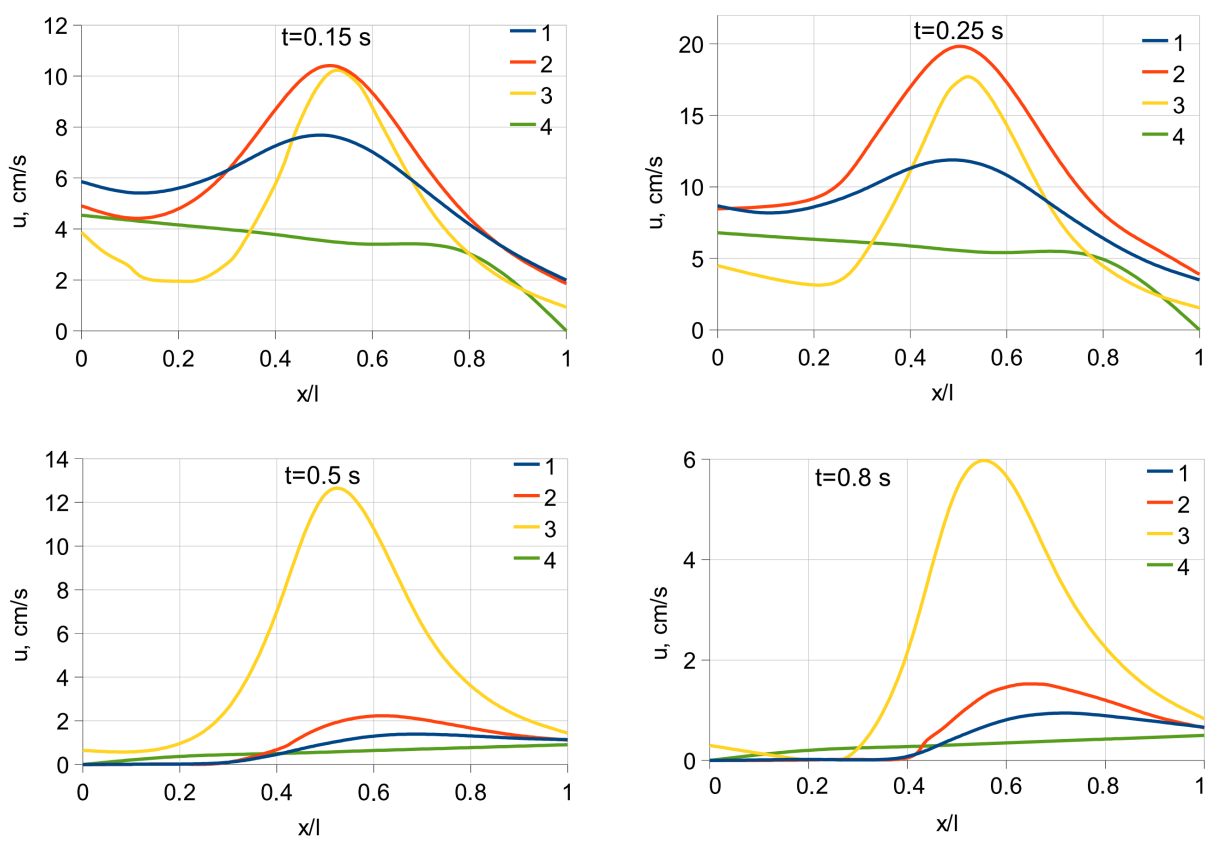

Figure 7: Velocity profiles in artery 2 with plaque 1 ( $1-50 \%$ lumen, $2-30 \%$ lumen, $3-10 \%$ lumen, 4 - healthy vessel) at $0.15,0.25,0.5,0.8$ seconds after the beginning of the cardiac cycle.
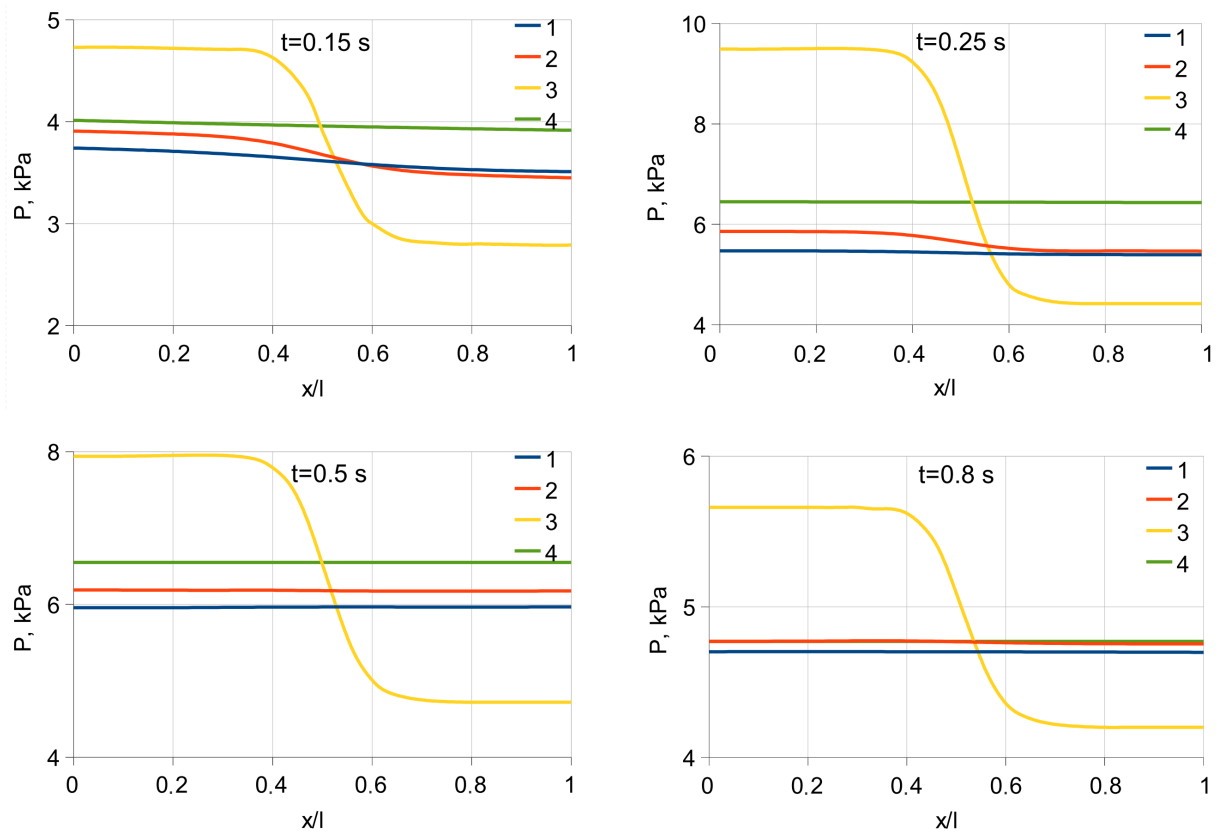

Figure 8: Pressure profiles in artery 2 with plaque 2 ( $1-50 \%$ lumen, $2-30 \%$ lumen, $3-10 \%$ lumen, 4 - healthy vessel) at $0.15,0.25,0.5,0.8$ seconds after the beginning of the cardiac cycle. 

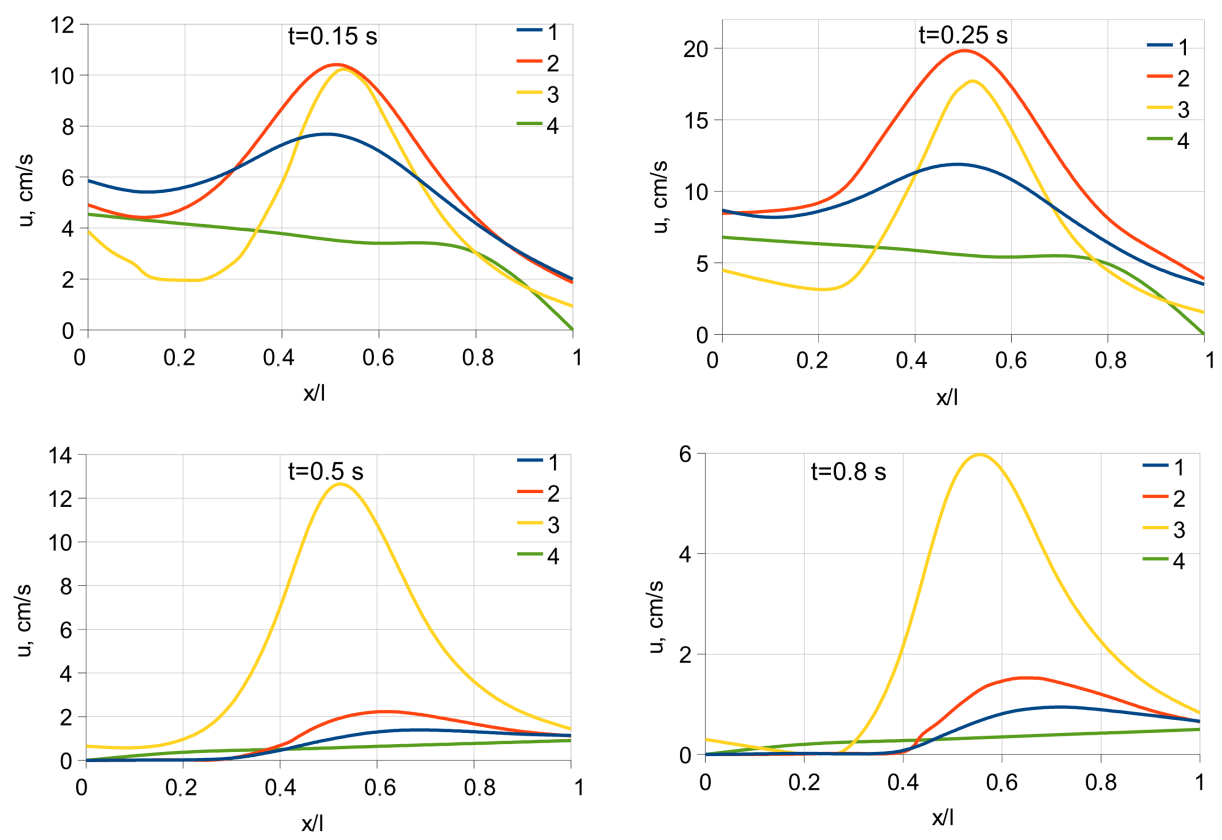

Figure 9: Velocity profiles in artery 2 with plaque 2 ( $1-50 \%$ lumen, $2-30 \%$ lumen, $3-10 \%$ lumen, 4 - healthy vessel) at $0.15,0.25,0.5,0.8$ seconds after the beginning of the cardiac cycle.
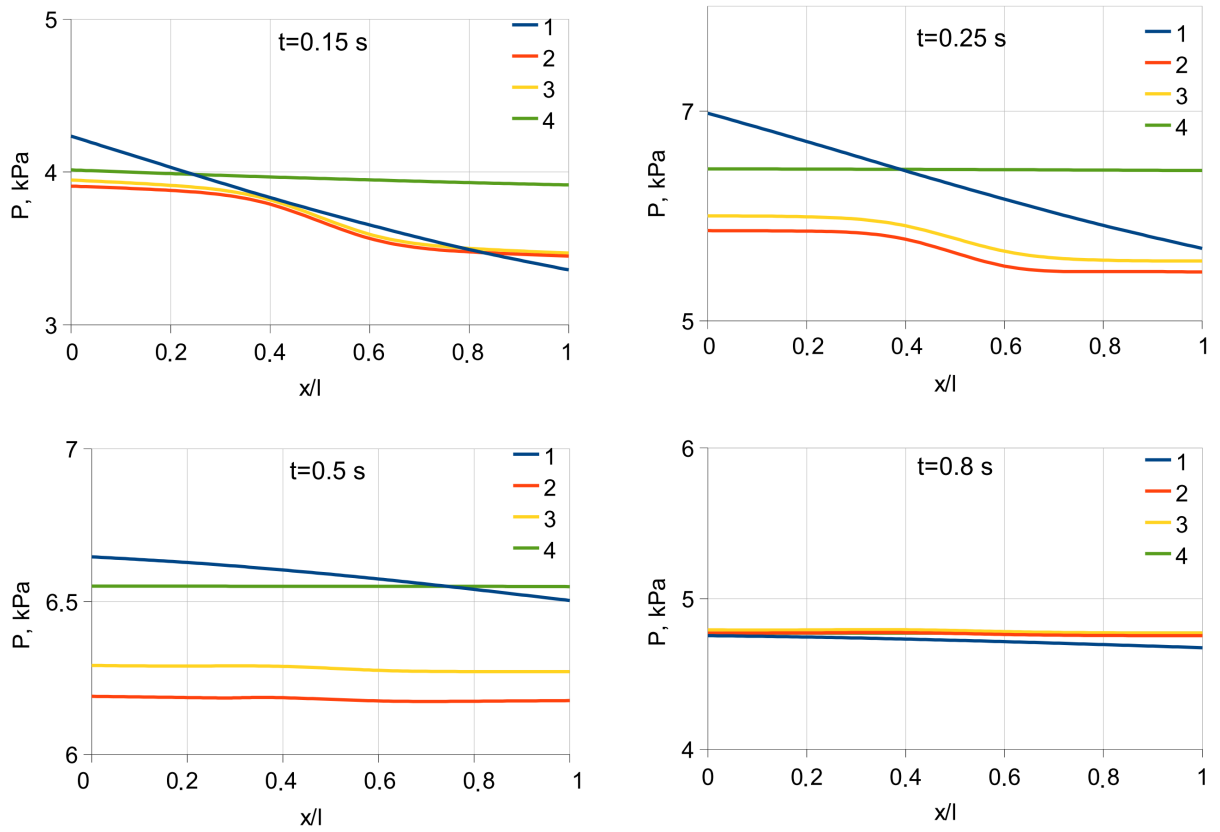

Figure 10: Pressure profiles in artery 2 with plaque of lumen $30 \%$ ( 1 - type 1, 2 - type 2, 3 type 3,4 - healthy vessel) at $0.15,0.25,0.5,0.8$ seconds after the beginning of the cardiac cycle. 

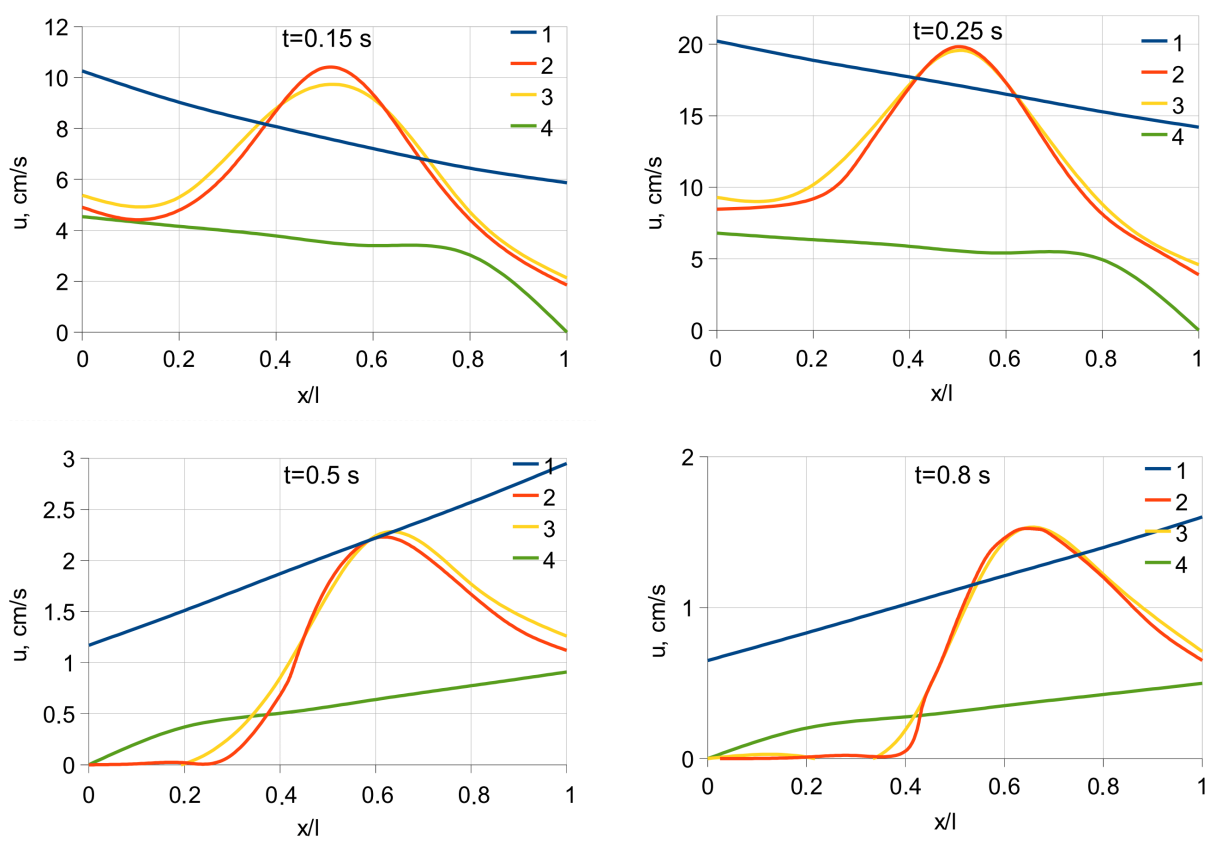

Figure 11: Velocity profiles in artery 2 with plaque of lumen $30 \%$ ( 1 - type 1,2 - type $2,3-$ type 3,4 - healthy vessel) at $0.15,0.25,0.5,0.8$ seconds after the beginning of the cardiac cycle.

junction and thus the dominant velocities are positive there. The comparison shows that in distal vessels both velocity and pressure are increased while in proximal vessels pressure is increased and velocity is decreased. Such stagnation region is potentially attractive for new plaque formation.

\section{Discussion}

Obviously, 2D models can't describe 3D material properties such as residual stresses, throughthickness stress distributions. It is also well-known that soft biological tissues exhibit a nonlinear behavior especially at high stresses. Our linear isotropic model is an approximation and the first step for the (fig. 2) as one of the basic cases. It may seems little in common with real plaques geometry. This simplified description allows us to calibrate and validate parameters of the general computational model of the wall elasticity via comparison to the analytical solution provided in sec. 2.3. The practical importance of this approximation is raised up when network haemodynamics is considered and main attention is paid to the regional and global effects as is in the numerical experiment desribed by the figs. 12-16. rather then to the local ones.

\section{Conclusions and future directions}

We presented the new methodology of coupling the network global haemodynamics and elastic wall response for the diseased vessels. The advantage of our approach is that it allows us to study 
$P$, vessel 48

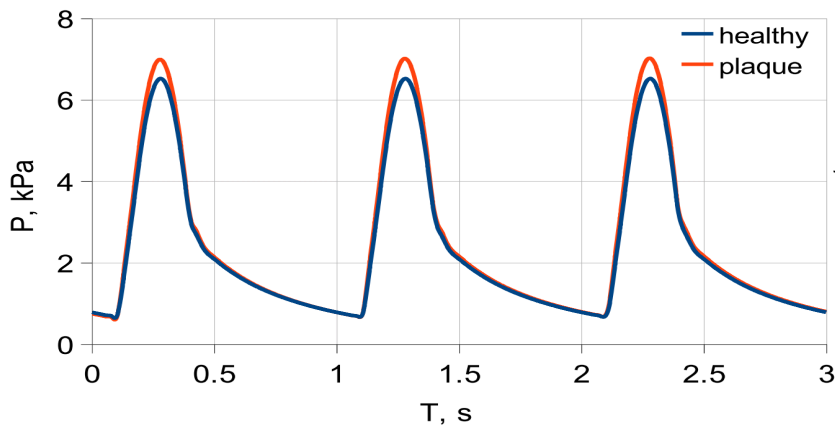

$U$, vessel 48

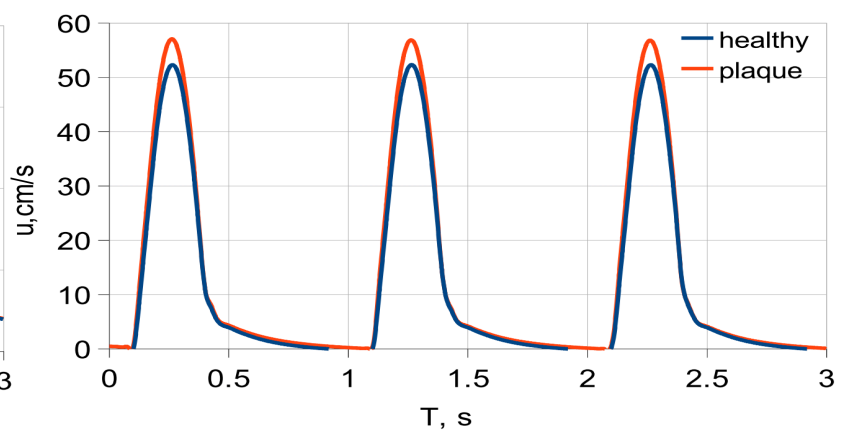

Figure 12: Regional blood flow redistribution: vessel 48.

$P$, vessel 49

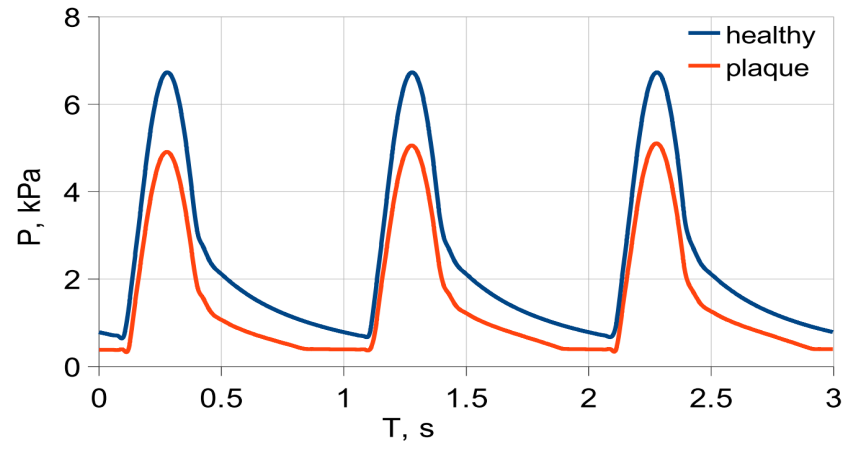

U, vessel 49

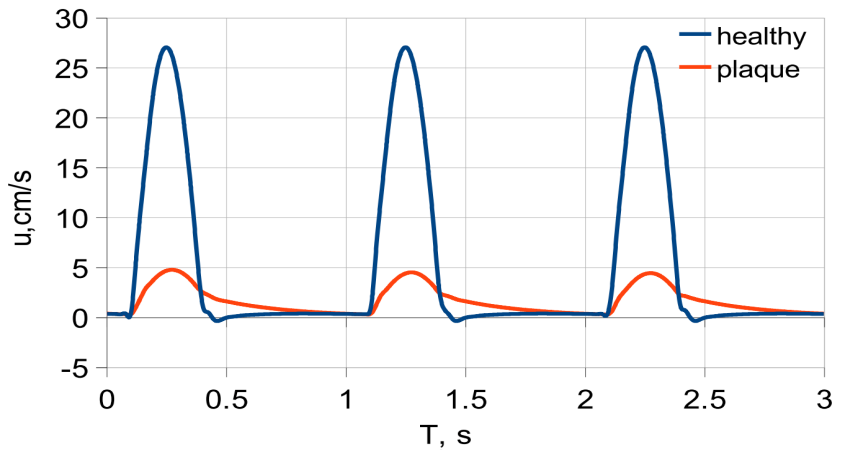

Figure 13: Regional blood flow redistribution: vessel 49.

$P$, vessel 50

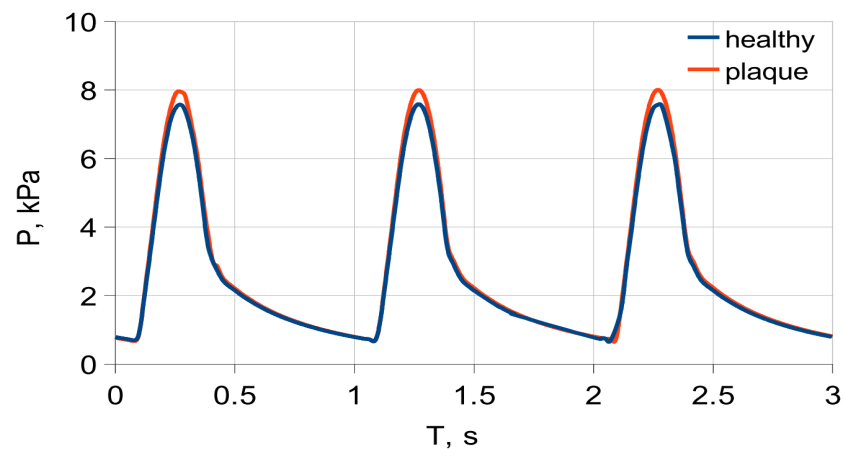

U, vessel 50

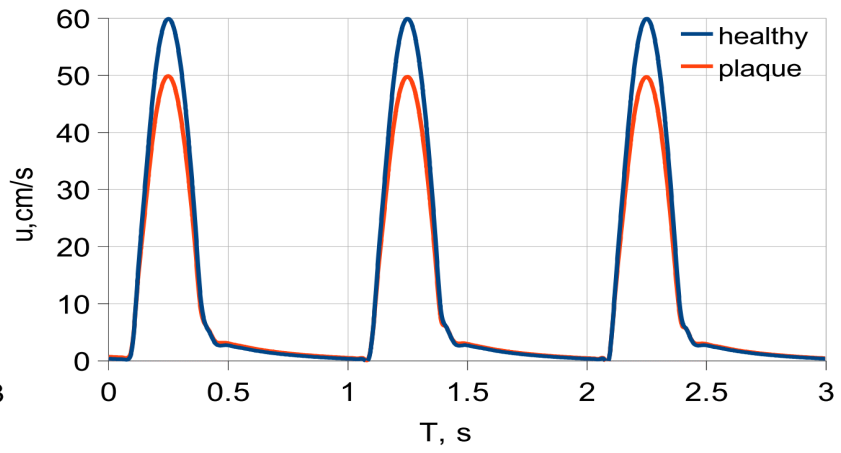

Figure 14: Regional blood flow redistribution: vessel 50. 

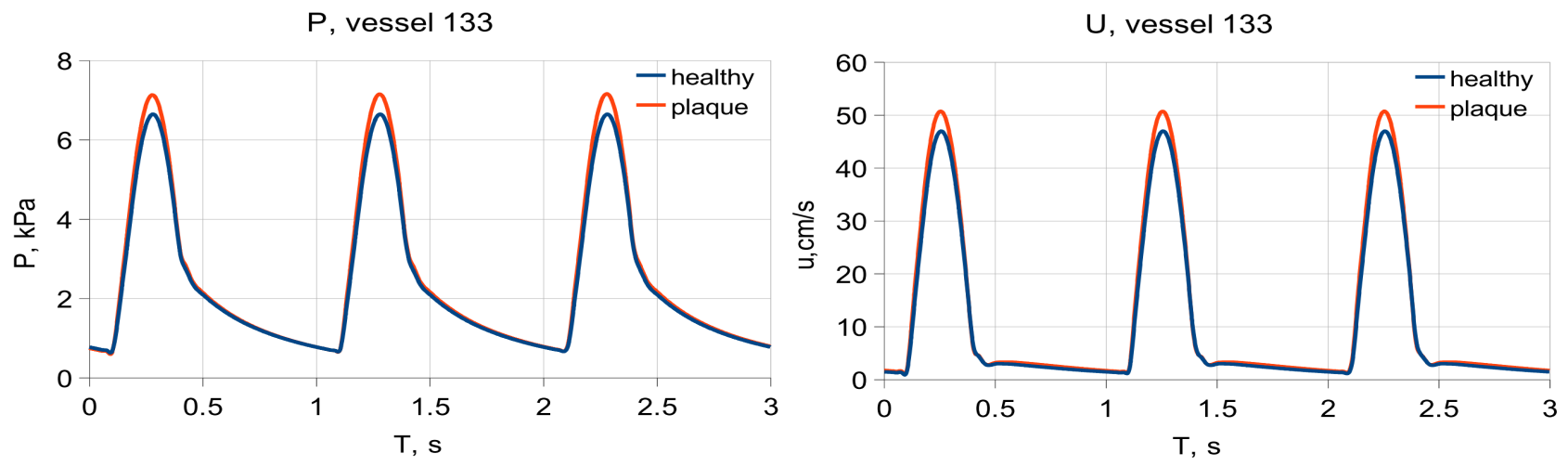

Figure 15: Regional blood flow redistribution: vessel 133.

$P$, vessel 103

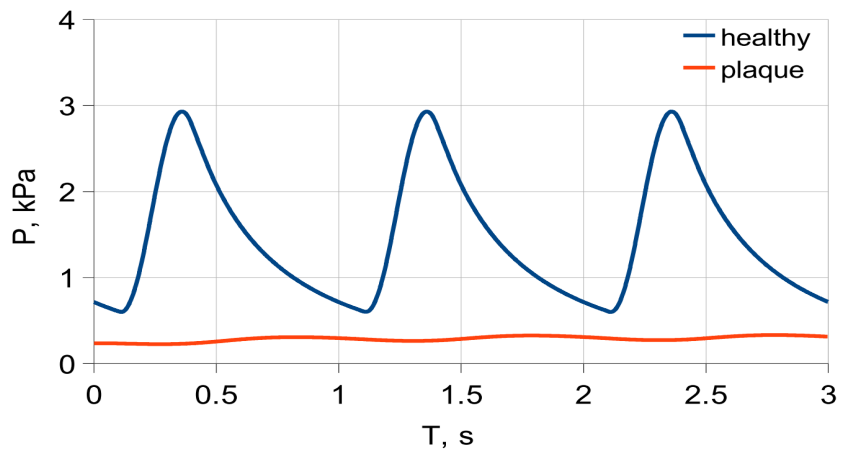

U,vessel103

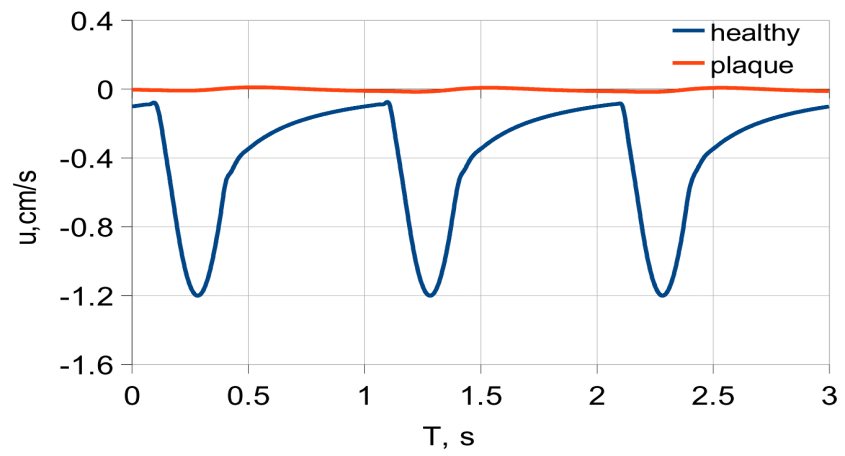

Figure 16: Regional blood flow redistribution: vessel 103.

regional and global changes in the blood circulation caused by pathalogical changes of vessel walls and to detect the diseased region on the basis of experimental data from the ultra Doppler measurements. The combined model can be used as a tool for the clinicians analyzing various scenarios of intravascular intervention and development of vascular diseases such as atherosclerosis.

We analyzed the regional blood flow redistribution only for a small part of the systemic circulation. Our future work will be focused on the numerical study of the full systemic network with distributed non-adjacent diseased vessels.

\section{Acknowledgements}

This work was partially supported by the Federal Program "Scientific and pedagogical staff of innovative Russia", RFBR grants 08-01-00159, 09-01-00115, 10-01-91055, 11-01-00971, 11-0100855 . 


\section{References}

[1] G. C. Cheng, H. M. Loree, R. D. Kamm, M. C. Fishbein, R. T. Lee Distribution of circumferential stress in ruptured and stable atherosclerotic lesions. A structural analysis with histopathological correlation. Circ. Res., 4 (1993), No. 87, 1179-1187.

[2] L. Formaggia, A. Quarteroni, A. Veneziani. Cardiovascular mathematics. Heidelberg, DE: Springer, 2009.

[3] T. C. Gasser, G. A. Holzapfel. A rate-independent elastoplastic constitutive model for biological fiber-reinforced composites at finite strains: continuum basis, algorithmic formulation and finite element implementation. Comp. Mech., 29 (2002), No. 4-5, 340-360.

[4] G. A. Holzapfel, T. C. Gasser, R. W. Ogden. A new constitutive framework for arterial wall mechanics and a comparative study of material models. J. Elasticity, 61 (2000), No. 1, 1-48.

[5] G. A. Holzapfel, M. Stadler, C. A. Schulze-Bauer. A layer-specific three-dimensional model for the simulation of balloon angioplasty using magnetic resonance imaging and mechanical testing. Ann. Biomed. Eng., 30 (2002), No. 6, 753-767.

[6] N. El Khatib, S. Génieys, V. Volpert. Atherosclerosis initiation modeled as an inflammatory process. Math. Model. Nat. Phen., 2 (2007), No. 2, 126-141.

[7] N. El Khatib, S. Génieys, A. M. Zine, V. Volpert. Non-Newtonian effects in a fluid-structure interaction model for atherosclerosis. J. Tech. Phys., 1 (2009), No. 50, 55-64.

[8] H. M. Loree, R. D. Kamm, R. G. Stringfellow, R. T. Lee. Effects of fibrous cap thickness on peak circumferential stress in model atherosclerotic vessels. Circ. Res., 4 (1992), No. 71, 850-858.

[9] Y. Mori, C. S. Peskin A universal programmable fiber architecture for the representation of a general incompressible linearly elastic material as a fiber-reinforced fluid Adv. Appl. Math., 43 (2009), No. 1, 75-100.

[10] T. J. Pedley, X. Y. Luo. Modelling flow and oscillations in collapsible tubes. Theor. Comp. Dluid Dyn., 10 (1998), No. 1-4, 277-294.

[11] M. Rosar, C. Peskin. Fluid flow in collapsible elastic tubes: a three-dimensional numerical model. New York J. Math., 7 (2001), 281-302.

[12] S. S. Simakov, A. S. Kholodov. Computational study of oxygen concentration in human blood under low frequency disturbances. Mat. Mod. Comp. Sim., 1 (2009), No. 283-295.

[13] C. A. Taylor, M. T. Draney. Experimental and computational methods in cardiovascular fluid mechanics. Ann. Rev. Fluid Mech., (2004), No. 36, 197-231. 
[14] C. Tu, C. Peskin. Stability and instability in the computation of flows with moving immersed boundaries: a comparison of three methods. SIAM J. Scientific and Statistical Computing, 6 (1992), No. 13, 1361-1376.

[15] F. N. Van de Vosse. Mathematical modelling of the cardiovascular system. J. Eng. Math., (2003), No. 47, 175-183.

[16] Y. V. Vassilevski, S. S. Simakov, S. A. Kapranov. A multi-model approach to intravenous filter optimization. Int. J. Num. Meth. Biomed. Eng., 26 (2010), No.7, 915-925. 